\title{
TEORES DE CLOROFILA DETERMINADOS POR MEDIDOR PORTÁTIL E SUA RELAÇÃO COM FORMAS DE NITROGÊNIO EM FOLHAS DE TOMATEIRO CULTIVADOS EM DOIS TIPOS DE SOLO ${ }^{(1)}$
}

\author{
TADEU GRACIOLLI GUIMARÃES (2), PAULO CESAR REZENDE FONTES (3,6), PAULO ROBERTO \\ GOMES PEREIRA ${ }^{(2)}$, VICTOR H. ALVAREZ V. ${ }^{(4,6)} \&$ PEDRO HENRIQUE MONNERAT ${ }^{(5)}$
}

\begin{abstract}
RESUMO
O presente estudo objetivou determinar os teores de clorofila no limbo foliar de tomateiros submetidos a doses crescentes de nitrogênio $(\mathrm{N})$, por meio de medidor portátil de clorofila SPAD-502 e por método convencional de laboratório, estabelecer a relação entre esses valores e relacioná-los com os teores de $\mathrm{N}$ na planta. Plantas de tomateiro (Lycopersicon esculentum Mill.), cv. Santa Clara, cultivadas em vasos contendo amostras de um solo de textura argilosa classificado como Podzólico Vermelho-Amarelo Câmbico (PVC) e de um solo de textura areia franca classificado como Areia Quartzosa (AQ), foram submetidas a cinco doses de $\mathrm{N}\left(25,125,225,325\right.$ e $\left.425 \mathrm{mg}^{-\mathrm{dm}^{-3}}\right)$, aplicadas parceladamente, via solução nutritiva. No início da antese do terceiro cacho, determinaram-se as concentrações de clorofila no limbo da folha oposta a ele por meio de medidor portátil de clorofila SPAD-502 e pelo método padrão de Arnon. Determinaram-se as concentrações de $\mathrm{N}-\mathrm{NO}_{3}$ na seiva e na matéria seca do pecíolo, e as de $\mathrm{N}-\mathrm{NO}_{3}$, N-orgânico e N-total na matéria seca do limbo. As concentrações de clorofila, avaliadas por ambos os métodos, aumentaram significativamente
\end{abstract}

\footnotetext{
(1) Parte da Tese de Doutorado do primeiro autor. Recebido para publicação em 14 de agosto de 1998 e aceito em 25 de março de 1999.

(2) Universidade Federal de Uberlândia, Departamento de Agronomia, Câmpus Umuarama, 38400-902 Uberlândia (MG).

(3) Universidade Federal de Viçosa, Departamento de Fitotecnia, 36571-000 Viçosa (MG).

(4) Universidade Federal de Viçosa, Departamento de Solos.

(5) Universidade Estadual do Norte Fluminense, Laboratório de Fitotecnia/CCTA, 28015-620 Campos (RJ).

${ }^{(6)}$ Com bolsa de produtividade em pesquisa do CNPq.
} 
com o acréscimo nas doses de $\mathrm{N}$, nos dois solos. Os níveis críticos de clorofila obtidos pelo método padrão foram 77,3 e 83,6 $\mu \mathrm{g}$. $\mathrm{cm}^{-2}$ de limbo foliar para PVC e AQ respectivamente, enquanto, com o medidor portátil, foram de 45,5 e 43,8 unidades SPAD, para o PVC e a AQ respectivamente. $\mathrm{O}$ método do medidor SPAD apresentou correlações maiores que as obtidas para o de laboratório, seja com a produção de matéria seca da parte aérea, seja com as formas de $\mathrm{N}$ analisadas. Os dois métodos de determinação de clorofila apresentaram maiores correlações com as concentrações de $\mathrm{N}^{-\mathrm{NO}_{3}}$ na seiva do PVC, e com as concentrações de N-org e $\mathrm{N}$-total na matéria seca do limbo na AQ. A estimativa das concentrações de clorofila nas folhas do tomateiro, expressas na forma padrão $(\mathrm{CP})$, a partir das leituras obtidas no medidor $\mathrm{SPAD}$, permitiu o ajuste de equação linear $\left(\mathrm{CP}=-77,233+3,54725^{* *} \mathrm{SPAD} \mathrm{R}^{2}=0,766\right)$.

Termos de indexação: tomateiro, Lycopersicon esculentum, clorofila, nitrogênio, nível crítico, método convencional e medidor portátil.

\title{
ABSTRACT \\ RELATIONS AMONG CHLOROPHYLL CONTENTS DETERMINED BY A PORTABLE METER AND NITROGEN FORMS IN LEAVES OF TOMATOES CULTIVATED IN TWO SOIL TYPES
}

\begin{abstract}
This work aimed to determine chlorophyll concentrations of tomato (Lycopersicon esculentum Mill.) plants cv. Santa Clara fertilized with five nitrogen (N) rates by means of conventional laboratory methodology and by the SPAD-502 portable chlorophyll meter. The relationship between chlorophyll and $\mathrm{N}$ values was determined by these two methods. Tomato plants were grown in pots with a clay soil classified as Cambic Red-Yellow Podsol (CRP) and a sand-course soil classified as Quartzic Sand (QS), and received five N rates (25, $125,225,325$ and $425 \mathrm{mg}$. $\mathrm{dm}^{-3}$ ) sidedressed as nutrient solutions. At the onset of $3^{\text {rd }}$ cluster anthesis, chlorophyll concentrations were determined in leaves opposite to this cluster by the portable chlorophyll meter SPAD-502 and at laboratory by Arnon's methodology. $\mathrm{NO}_{3}-\mathrm{N}$ was determined in petiole sap and in dry matter, while $\mathrm{NO}_{3}-\mathrm{N}$, org- $\mathrm{N}$ and total-N were determined in leaf blade dry matter. Chlorophyll concentrations increased with $\mathrm{N}$ rates irrespective of method of determination, in both soils. Critical levels in soils CRP and QS were, respectively, 77.3 and $83.6 \mu \mathrm{g}$. $\mathrm{cm}^{-2}$ by laboratory methodology and 45.5 and $43.8 \mathrm{SPAD}$ units by the portable meter. Correlation coefficients of chlorophyll concentrations with top dry weight and $\mathrm{N}$ forms were greater for the SPAD method. Both methods of chlorophyll determination presented greatest correlation with sap $\mathrm{NO}_{3}-\mathrm{N}$ in soil CRP, and with org-N and total-N in soil QS. The estimative of chlorophyll concentrations (laboratory method) by means of SPAD readings allowed the adjustment of a linear equation $\left(\mathrm{CP}=-77.233+3.54725^{* *}\right.$ SPAD $\left.R^{2}=0.766\right)$.
\end{abstract}

Index terms: tomatoes, Lycopersicon esculentum, chlorophyll, nitrogen, critical level, conventional laboratory methodology and portable meter.

\section{INTRODUÇÃ̃O}

Determinações periódicas e precisas do estado nutricional do tomateiro podem auxiliar no manejo da fertilização nitrogenada da cultura.
Para tal, determinam-se, geralmente, as concentrações de $\mathrm{N}$ na matéria seca de folhas, em condições de laboratório, empregando-se métodos analíticos demorados e de alto custo. 
Tem sido demonstrado que a concentração de clorofila pode indicar a concentração de $\mathrm{N}$ na folha de milho (Girardin et al., 1985), podendo ser uma determinação mais sensível às variações do suprimento de $\mathrm{N}$ que as determinações do elemento na matéria seca das folhas, o que possibilitaria maior rapidez na detecção da deficiência de N (Schepers et al., 1992).

O método padrão de determinação de clorofila em laboratório (Arnon, 1949), ainda que fácil, apresenta desvantagens, como coleta destrutiva do material vegetal, extração via maceração com acetona e leitura em espectrofotômetro. Com o advento de medidores portáteis (Yadava, 1986), a determinação de clorofila tor nou-se mais fácil e rápida, sendo realizada diretamente no campo. O modelo atual, SPAD-502, da Minolta Camera Co. Ltd., tem sido utilizado com sucesso para diagnosticar o estado nitrogenado de culturas como milho (Piekielek \& Fox, 1992; Blackmer et al., 1994; Smeal \& Zhang, 1994), batata (Minotti et al., 1994), trigo (Fox et al., 1994), entre outras, não tendo sido avaliado, porém, na determinação do teor de clorofila das folhas do tomateiro submetidas a doses crescentes de $\mathrm{N}$.

No presente trabalho, determinaram-se os teores de clorofila e de $\mathrm{N}$ em folha de tomateiros cultivados em vasos, em amostras de dois solos de diferentes texturas, na presença de doses crescentes de $\mathrm{N}$. Os teores de clorofila foram determinados por meio de método padrão de laboratório (Arnon, 1949) e pelo que utiliza o medidor portátil de clorofila SPAD-502. Objetivou-se determinar os níveis críticos de clorofila e relacioná-los com os dois métodos, e verificar a relação existente entre os teores de clorofila e de formas de $\mathrm{N}$ no limbo e no pecíolo foliar.

\section{MATERIAL E MÉTODOS}

O experimento foi realizado em casa de vegetação do Departamento de Fitotecnia da Universidade Federal de Viçosa (UFV), de 24/11/95 a 27/1/96, utilizando-se vasos de 12,0 L com $10 \mathrm{dm}^{3}$ de solo, contendo amostras de um solo argiloso, classificado como Podzólico Vermelho-Amarelo Câmbico (PVC) e de um solo areia franca, classificado como Areia Quartzosa (AQ). A amostra de solo argiloso proveio de uma área destinada ao cultivo de hortaliças em Viçosa, enquanto a de solo areia franca foi obtida em área sem exploração agrícola atual em Três Marias (MG). Tais amostras foram analisadas química e fisicamente nos Laboratórios de Fertilidade e de Física de Solos, do Departamento de Solos da UFV. As principais características das amostras PVC e AQ eram, respectivamente, argila (\%) 44 e 12; matéria orgânica (dag. $\left.\mathrm{kg}^{-1}\right)$ 1,58 e 0,46; capacidade de campo (kg. $\left.\mathrm{kg}^{-1}\right)$ 0,329 e 0,$065 ; \mathrm{pH}\left(\mathrm{em} \mathrm{H}_{2} \mathrm{O}\right) 5,5$ e 4,$7 ; \mathrm{P}\left(\mathrm{mg} . \mathrm{dm}^{-3}\right)$ 81,1 e 2,$1 ; \mathrm{K}^{+}\left(\mathrm{cmol}_{\mathrm{c}} \cdot \mathrm{dm}^{-3}\right) \quad 0,18$ e 0,$04 ; \mathrm{Ca}^{2+}$ $\left(\mathrm{cmol}_{\mathrm{c}} \cdot \mathrm{dm}^{-3}\right) 3,2$ e 0,$1 ; \mathrm{Mg}^{2+}\left(\mathrm{cmol}_{\mathrm{c}} \cdot \mathrm{dm}^{-3}\right)$ 0,7 e 0,0 ; $\mathrm{Al}^{3+}\left(\mathrm{cmol}_{\mathrm{c}} \cdot \mathrm{dm}^{-3}\right)$ 0,0 e 0,$3 ; \mathrm{H}+\mathrm{Al}\left(\mathrm{cmol}_{\mathrm{c}} \cdot \mathrm{dm}^{-3}\right) 2,7 \mathrm{e}$ 3,6; SB $\left(\mathrm{cmol}_{\mathrm{c}} \cdot \mathrm{dm}^{-3}\right) 4,08$ e 0,14 , e CTCt $\left(\mathrm{cmol}_{\mathrm{c}} \cdot \mathrm{dm}^{-3}\right)$ 6,78 e 3,74 .

$\mathrm{Na}$ calagem do solo $\mathrm{AQ}$, aplicou-se a mistura de $\mathrm{CaCO}_{3}$ e $\mathrm{MgO}$ (4:1 mol), ambos p.a., para elevar a saturação de bases a $60 \%$. A adubação fosfatada constou de $3 \mathrm{~g}$ de $\mathrm{P}$ por vaso no solo AQ e $5 \mathrm{~g}$ de $\mathrm{P}$ por vaso no PVC, na forma de superfosfato simples. Metade da dose de $\mathrm{P}$ foi aplicada em $1 \mathrm{dm}^{3}$ do volume do solo, o qual foi localizado superficialmente nos vasos, e metade, nos $9 \mathrm{dm}^{3}$ restantes.

Em seguida, no mesmo dia, os vasos foram irrigados e seis sementes de tomate (Lycopersicon esculentum Mill.), cultivar Santa Clara, foram semeadas. Após a emergência, foi feito desbaste, deixando-se uma planta por vaso.

Os tratamentos constaram de 5 doses de $\mathrm{N}$ (25, $125,225,325$ e $425 \mathrm{mg}$. $\left.\mathrm{dm}^{-3}\right)$, parceladas em seis aplicações em cobertura, via solução nutritiva ( $25 \%$ como $\mathrm{N}-\mathrm{NH}_{4}^{+}$), utilizando-se as fontes $\mathrm{NH}_{4} \mathrm{NO}_{3}$, $\mathrm{KNO}_{3}$ e $\mathrm{NaNO}_{3}$, todas p.a. As doses de $\mathrm{N}$ foram parceladas conforme o seguinte cronog rama, em dias após a semeadura (DAS): $5 \%$ em cada aplicação aos $14 \mathrm{e}$ $24 ; 10 \%$ aos $33 ; 20 \%$ aos 43 e $30 \%$ aos 49 e aos 54 . As unidades experimentais foram dispostas na estufa seguindo o delineamento de blocos casualizados, com cinco repetições. 
Empregaram-se $\mathrm{K}, \mathrm{Ca}, \mathrm{Mg}$, S e micronutrientes em cobertura, na forma de soluções nutritivas, sendo os micronutrientes também aplicados por via foliar. As aplicações de $\mathrm{K}$ e $\mathrm{S}$ foram realizadas conjuntamente com as de $\mathrm{N}$, seguindo o mesmo parcelamento, totalizando 182 e $40 \mathrm{mg}$. $\mathrm{dm}^{-3}$ de $\mathrm{K}$ e de $\mathrm{S}$ respectivamente. No solo AQ, usaram-se 0,975 e $0,45 \mathrm{cmol}_{\mathrm{c}} \cdot \mathrm{dm}^{-3}$ de $\mathrm{Ca}$ e de $\mathrm{Mg}$ respectivamente, e, no PVC, 0,75 e 0,3 $\mathrm{cmol}_{\mathrm{c}} \mathrm{dm}^{-3}$ de $\mathrm{Ca}$ e de $\mathrm{Mg}$ respectivamente. No PVC, as doses de micronutrientes, em mg.dm ${ }^{-3}$, totalizaram 2 de $\mathrm{Zn} ; 1$ de $\mathrm{B} ; 1$ de $\mathrm{Cu} ; 0,5$ de $\mathrm{Mn}$ e 0,2 de Mo, enquanto no solo AQ totalizaram 4 de $\mathrm{Zn} ; 1,5$ de B; 1,5 de $\mathrm{Cu} ; 1$ de Mn e 0,4 de Mo. No solo AQ, aplicaram-se maiores dosagens de $\mathrm{Ca}, \mathrm{Mg}$ e de micronutrientes em vista das condições de baixa fertilidade, menor teor de matéria orgânica e menor CTC potencial originalmente apresentadas por essa amostra de solo.

Foram feitas aplicações foliares de soluções de $\mathrm{H}_{3} \mathrm{BO}_{3}$ a 1,5 g. $\mathrm{L}^{-1}, \mathrm{ZnSO}_{4} \cdot 7 \mathrm{H}_{2} \mathrm{O}$ a 7,5 g. $\mathrm{L}^{-1} \mathrm{e}$ $\mathrm{Na}_{2} \mathrm{MoO}_{4} \cdot 2 \mathrm{H}_{2} \mathrm{O}$ a $0,125 \mathrm{~g} . \mathrm{L}^{-1}$ no aparecimento do primeiro cacho, aos 34 e aos 55 DAS.

Pulverizações com fungicida, inseticida e acaricida foram feitas sempre que necessárias.

$\mathrm{Na}$ abertura das flores do terceiro cacho, aos 57 DAS, determinaram-se os teores de clorofila na folha oposta a ele, entre as $7 \mathrm{e}$ as $9 \mathrm{~h}$, utilizando o medidor portátil de clorofila SPAD-502 (Minolta Camera Co. Ltd.), em cinco folíolos de cada folha, sendo dois de cada lado da folha e o terminal central. Das cinco leituras, calculou-se a média para cada folha amostrada, utilizando-se o próprio medidor. Imediatamente após o término das leituras, as folhas opostas ao terceiro cacho foram destacadas de cada planta, acondicionadas em sacos plásticos e, no laboratório, mantidas sob refrigeração. Em seguida, tomou-se, de cada folha amostrada, na base de um dos folíolos laterais usados para a leitura com o medidor, um disco de $0,9 \mathrm{~cm}$ de diâmetro, o qual foi macerado em acetona a $80 \%$, na presença de $\mathrm{CaCO}_{3}$. Os extratos obtidos foram filtrados através de papel-filtro rápido e coletados em balões volumétricos de $25 \mathrm{ml}$, completando-se o volume ao final da filtragem. A densi- dade ótica dos filtrados foi lida em espectrofotômetro a 645 e $663 \mathrm{~nm}$, utilizando cubetas de quartzo. A partir dessas leituras, determinou-se a concentração de clorofila (conc. cl.) nas soluções de leitura, por meio da fórmula abaixo, recomendada por Arnon (1949):

$$
\text { Conc. cl.: } \mu \mathrm{g} \cdot \mathrm{cm}^{-3}=20,2 \mathrm{~A}_{645 \mathrm{~nm}}+8,02 \mathrm{~A}_{663 \mathrm{~nm}}
$$

Posteriormente, os valores das concentrações de clorofila no limbo foliar foram transformados para $\mu \mathrm{g} . \mathrm{cm}^{-2}$ de clorofila.

As determinações das formas de $\mathrm{N}$ no tomateiro estão detalhadas em Guimarães et al. (1998) e, basicamente, constam dos procedimentos listados a seguir.

Nas folhas opostas ao terceiro cacho, retiraram-se seções de $1 \mathrm{~cm}$ de comprimento da base dos pecíolos e maceraram-nas em "espremedor de alho", para extração da seiva. Com o auxílio de uma micropipeta, a seiva foi coletada e analisada em medidor portátil de nitrato (C-141 Cardy Nitrate MeterHoriba, Inc.) equipado com microeletrodo sensível ao nitrato. Posteriormente, os pecíolos foram separados do limbo foliar, sendo ambos, separadamente, acondicionados em sacos de papel, secos em estufa de circulação forçada de ar a $75^{\circ} \mathrm{C}$ por 72 horas, pesados, passados por moinho tipo Wiley com peneira de 20 mesh e armazenados. Nas matérias secas do pecíolo e do limbo, após a extração com água em banho-maria a $45^{\circ} \mathrm{C}$, durante uma hora, determinaram-se as concentrações de $\mathrm{N}-\mathrm{NO}_{3}$ por colorimetria, em espectrofotômetro a $410 \mathrm{~nm}$ (Cataldo et al.,1975). Determinaram-se, ainda, após digestão sulfúrica, os teores de $\mathrm{N}$-orgânico (N-org.) na matéria seca do limbo por meio do reagente de Nessler (Jackson, 1982), calculando-se, posteriormente, os valores de $\mathrm{N}$-total (N-tot) a partir da soma dos teores de $\mathrm{N}-\mathrm{NO}_{3}$ e de N-orgânico.

As plantas tiveram seus pontos de crescimento eliminados imediatamente abaixo da quarta inflorescência, por ocasião do seu surgimento. Aos 64 DAS, foram cortadas rente ao solo, divididas em folhas, caules e frutos, os quais foram secos em estufa de circulação forçada de ar a $75^{\circ} \mathrm{C}$ por 72 horas, para posterior determinação da produção de matéria seca. 
Os dados foram submetidos a análises da variância e de regressão, ajustando-se equações de regressão relacionando as concentrações de clorofila obtidas às doses de nitrogênio aplicadas. A partir da dose de $\mathrm{N}$ necessária para obtenção de $99 \%$ da produção máxima de matéria seca da parte aérea, calculou-se o nível crítico de clorofila, para cada método de determinação (padrão de Arnon e medidor portátil de clorofila), nas plantas cultivadas em cada amostra de solo. As concentrações de clorofila foram relacionadas com a produção de matéria seca da parte aérea e com as concentrações de $\mathrm{N}-\mathrm{NO}_{3}$ na seiva e na matéria seca do pecíolo, e com as concentrações de $\mathrm{N}$-org e N-tot na matéria seca do limbo foliar por meio de correlação linear de Pearson.

Estudou-se a relação existente entre os valores resultantes dos dois métodos de determinação de clorofila, ajustando-se equações de regressão nas quais as concentrações de clorofila no limbo, obtidas pelo método padrão, foram tomadas como variável dependente e os valores obtidos com o medidor portátil, como variável independente. Os coeficientes de correlação e de determinação foram testados a $1(* *)$ e a $5 \%$ (*) de significância.

\section{RESULTADOS E DISCUSSÃO}

Os valores estimados máximos para a produção de matéria seca de frutos e da parte aérea das plantas cultivadas no solo PVC foram de 37,7 e 141,3 g. planta $^{-1}$ respectivamente, obtidos com a maior dose estudada, $425 \mathrm{mg}$. dm${ }^{-3}$ de N (Figura 1). Tal fato indica que, para esse solo, a fim de maximizar o crescimento, deveria ter sido aplicada dose de $\mathrm{N}$ ainda maior ou, talvez, ter modificado seu parcelamento. No solo $\mathrm{AQ}$, os valores estimados máximos foram de 23,6 e 95,7 g. vaso $^{-1}$ para a matéria seca de frutos e da parte aérea respectivamente, valores obtidos com 300 e 350 mg. $\mathrm{dm}^{-3}$ de $\mathrm{N}$ respectivamente (Figura 1). As doses necessárias para obter $99 \%$ da máxima produção de matéria seca da parte aérea foram de 404 e $301 \mathrm{mg}$. $\mathrm{dm}^{-3}$ de N, para os solos PVC e AQ respectivamente.

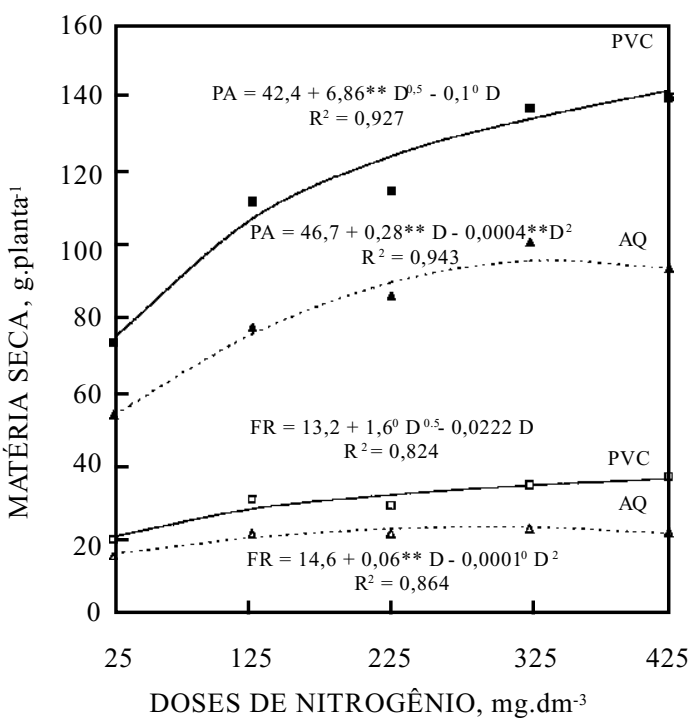

Figura 1. Produção de matéria seca de frutos (FR) e da parte aérea (PA) de tomateiros cultivados nos solos PVC (-) e AQ (---), em função de doses de $\mathbf{N}$.

As concentrações de clorofila na folha oposta ao terceiro cacho, determinadas por ambos os métodos, cresceram linearmente com o aumento das doses de $\mathrm{N}$ nos dois solos (Figuras 2 e 3). Lopez-Cantarero et al. (1994), utilizando o método padrão de laboratório, observaram que o aumento no fornecimento de $\mathrm{N}$ ao solo produziu aumento nas concentrações de clorofila total no limbo foliar de berinjela (Solanum melongena $\mathrm{cv}$. Bonica), espécie da mesma família do tomateiro. Similarmente, trabalhando com variedades de batata, também uma solanácea, Minotti et al. (1994) observaram elevação na concentração de clorofila, determinada com o medidor SPAD, com o aumento das doses de $\mathrm{N}$ aplicadas ao solo.

As concentrações de clorof ila nas plantas cultivadas no solo PVC variaram de 50 a $82,6 \mu \mathrm{g} . \mathrm{cm}^{-2}$ de limbo foliar, pelo método padrão, de 35 a 45,2 unidades SPAD, pelo portátil, enquanto, para o solo $\mathrm{AQ}$, variaram de 51,8 a $90,3 \mu \mathrm{g} . \mathrm{cm}^{-2}$ de limbo foliar e 35,5 a 46,5 unidades SPAD respectivamente (Figuras 2 e 3). Os níveis críticos obtidos pelo método padrão no PVC e na AQ foram de 77,3 e 83,6 $\mu \mathrm{g} . \mathrm{cm}^{-2}$ 


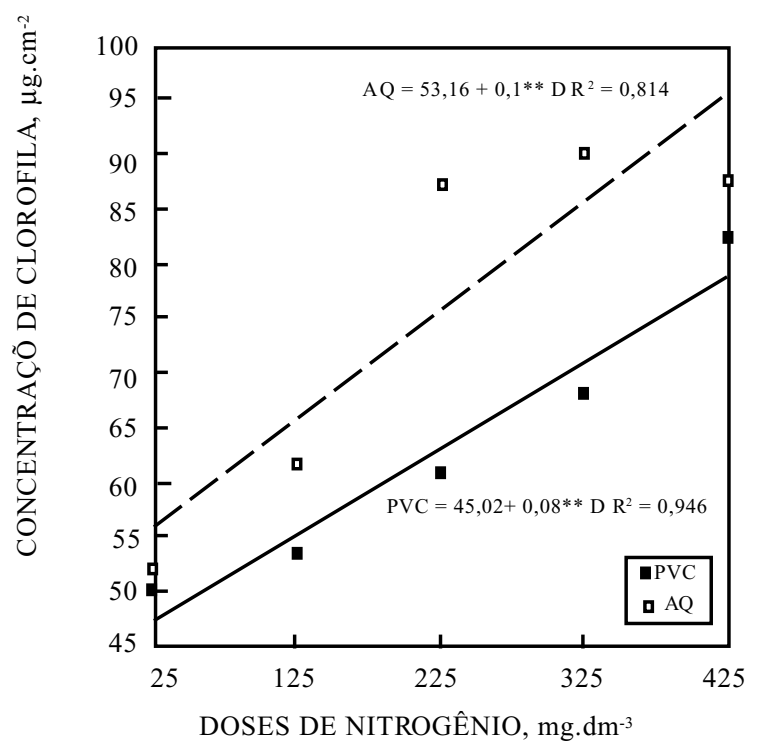

Figura 2. Concentração de clorofila determinada pelo método padrão no limbo foliar de tomateiros cultivados nos solos PVC (-) e AQ (---), em função de doses de $\mathbf{N}$.

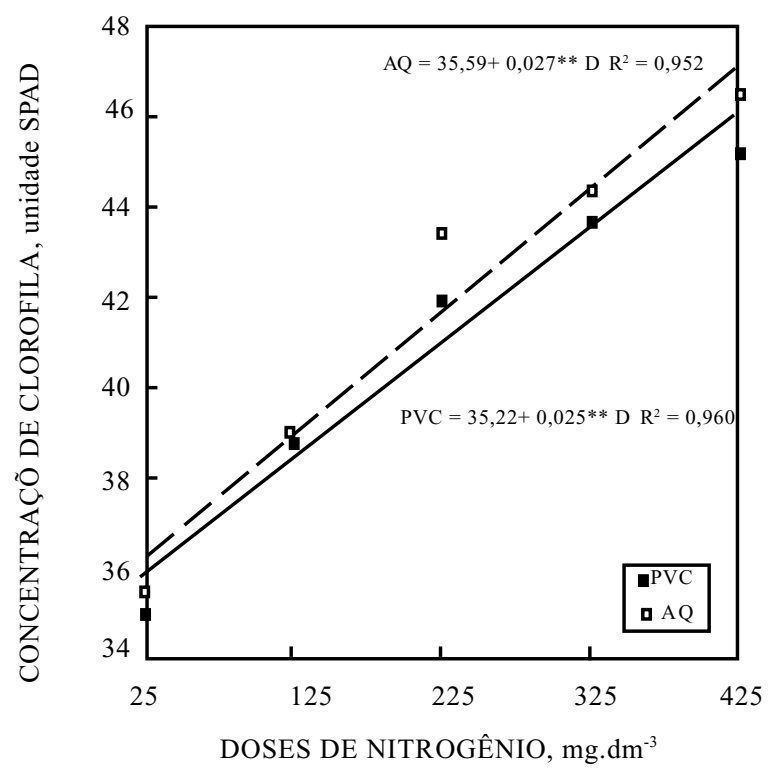

Figura 3. Concentração de clorofila determinada pelo medidor de clorofila SPDA-502 no limbo de tomateiros cultivados nos solos PVC (-)e AQ (---), em função de doses de $\mathbf{N}$. respectivamente, enquanto, pelo medidor SPAD, foram de 45,5 e 43,8 unidades SPAD, nos solos PVC e $A Q$ respectivamente. Os valores críticos obtidos com o medidor se assemelham ao obtido para milho (43,4 unidades SPAD) por Piekielek \& Fox (1992), diferindo, porém, do alcançado por Smeal \& Zhang (1994) para a mesma cultura, que foi de 52 unidades SPAD e, também, da faixa de valores (49-56 unidades SPAD) obtida por Minotti et al. (1994) para a batata. Deve-se ressaltar que esses resultados provêm de pesquisas realizadas em condições de campo, envolvendo outras espécies e diferentes épocas de amostragem.

As concentrações de clorofila determinadas pelos dois métodos, nos dois solos, apresentaram correlação positiva e significativa com a produção de matéria seca da parte aérea, sendo de $0,627 * *$ (PVC) e $0,750 * *$ (AQ) quando se utilizou o método padrão de Arnon, e de $0,823^{* *}$ (PVC) e $0,775^{* *}$ (AQ) quando se empregou o medidor SPAD. Esses resultados são corroborados com observações relatadas por Minnoti et al. (1994) e Blackmer \& Schepers (1995). Os coeficientes de correlação obtidos com os dados do medidor SPAD foram maiores que aqueles com o método tradicional, em ambos os solos, indicando que, além de ser mais rápido e de mais fácil execução, mostrou maior capacidade preditiva que o método padrão utilizado.

As correlações entre as concentrações de clorofila, determinadas com o medidor SPAD e as formas de $\mathrm{N}$ foram sempre maiores que as obtidas com o método padrão, em ambos os solos (Quadro 1), indicando que o medidor representa melhor alternativa para se inferir sobre o estado nitrogenado do tomateiro. No PVC, as maiores correlações foram verificadas com os teores de $\mathrm{NO}_{3}$ na seiva do pecíolo, enquanto para o AQ, as maiores correlações foram observadas com as formas de N-org e N-total (Quadro 1), fato que também foi verificado por Smeal \& Zhang (1994), para o milho.

As concentrações de clorofila, determinadas pelos dois métodos, não apresentaram correlações signif icativas com a concentração de N-org e N-total no limbo das plantas cultivadas no PVC (Quadro 1). 
Quadro 1. Correlações lineares de Pearson (r) entre as concentrações de clorofila no limbo foliar determinadas pelo método padrão de Arnon (CP) e pelo medidor portátil SPAD-502 (CS), e as formas de $\mathbf{N}$ em tomateiros cultivados nas amostras de solos PVC e AQ

\begin{tabular}{|c|c|c|c|c|}
\hline \multirow{2}{*}{ Formas de nitrogênio } & \multicolumn{2}{|c|}{$\mathrm{PVC}$} & \multicolumn{2}{|c|}{$\mathrm{AQ}$} \\
\hline & $\mathrm{CP}$ & $\mathrm{CS}$ & $\mathrm{CP}$ & $\mathrm{CS}$ \\
\hline $\mathrm{N}-\mathrm{NO}_{3}\left(\mathrm{mg}^{-\mathrm{L}^{-1}}\right) \ldots \ldots .$. & $0,502 * *$ & $0,530 * *$ & $0,483 * *$ & $0,697 * *$ \\
\hline $\mathrm{N}-\mathrm{NO}_{3}\left(\mathrm{mg} . \mathrm{kg}^{-1}\right) \ldots \ldots$. & 0,325 & $0,410^{*}$ & 0,310 & $0,607 * *$ \\
\hline N-org $\left(\mathrm{mg} . \mathrm{kg}^{-1}\right) \ldots \ldots \ldots$ & 0,252 & 0,328 & $0,630 * *$ & $0,802 * *$ \\
\hline N-total $\left(\mathrm{mg} . \mathrm{kg}^{-1}\right) \ldots \ldots$ & 0,262 & 0,336 & $0,630 * *$ & $0,804 * *$ \\
\hline
\end{tabular}

** e * = Significância a 1 e a $5 \%$ de probabilidade respectivamente.

Shadchina \& Dmitrieva (1995) também não encontraram correlação entre concentração de clorofila determinada pelo método padrão de Arnon e a concentração de $\mathrm{N}$ total no limbo de folhas de trigo.

A relação entre as concentrações de clorofila obtidas em laboratório pelo método padrão (CP) e as leituras obtidas com o medidor SPAD permitiu o ajuste de equação linear e significativa $(\mathrm{CP}=-77,233+$ $\left.3,54725^{* *} \mathrm{SPAD} \mathrm{R}^{2}=0,766\right)$, indicando que as leituras obtidas com o medidor podem ser utilizadas para estimar as concentrações de clorofila determinadas pelo método padrão. Em análise semelhante, Yadava (1986) obteve, para um conjunto de 22 espécies pertencentes a um total de 14 famílias, $\mathrm{R}^{2}=0,478$. O autor salientou, porém, que valores de $\mathrm{R}^{2}$ maiores poderiam ser obtidos caso a pesquisa tivesse envolvido um conjunto menor de espécies relacionadas entre si, como na de Gratani (1992), que estudou essa relação em um conjunto de seis espécies lenhosas, obtendo $\mathrm{R}^{2}=0,740$.

\section{CONCLUSÕES}

Em condições de vasos, principalmente com o solo AQ, no qual foi possível obter o ponto de máximo para a produção de matéria seca da parte aérea dentro do intervalo experimental utilizado, o medidor portátil de clorofila SPAD-502 representou alternativa para avaliar o teor de clorof ila do limbo foliar e, possivelmente, diagnosticar o estado de $\mathrm{N}$ do tomateiro.

\section{REFERÊNCIAS BIBLIOGRÁFICAS}

ARNON, D.I. Copper enzymes in isolated chloroplasts: polyphenoloxydase in Beta vulgaris. Plant Physiology, Maryland, 24(1):1-15, 1949.

BLACKMER, T.M. \& SCHEPERS, J. S. Use of a chlorophyll meter to monitor nitrogen status and schedule fertigation for corn. Journal of Production Agriculture, Madison, 8(1):56-60, 1995.

BLACKMER, T.M.; SCHEPERS, J. S. \& VARVEL, G. E. Light reflectance compared with other nitrogen stress measurements in corn leaves. Agronomy Journal, Madison, 86(6):934-938, 1994.

CATALDO, D.A.; HAROON, M.; SCHRADER, L.E. \& YOUNES, V.L. Rapid colorimetric determination of nitrate in plant tissue by nitration of salicilic acid. Communications in Soil Science and PlantAnalysis, New York, 6(1):71-80, 1975.

FOX, R.H., PIEKIELEK, W.P. \& MACNEAL, K.M. Using a chlorophyll meter to predict nitrogen fertilizer needs of winter wheat. Communications in Soil Science and Plant Analysis, New York, 25(3/4):171-181, 1994.

GIRARDIN, P.; TOLLENAAR, M. \& MULDON, J.F. The effect of temporary $\mathrm{N}$ starvation on leaf photosyntetic rate and chlorophyll content of maize. Canadian Journal of Plant Science, Ottawa, 65(3):491-500, 1985.

GUIMARÃES, T.G.; FONTES, P.C.R.; PEREIRA, P.R.G.; ALVAREZ V., V.H. \& MONNERAT, P.H. Determinação dos teores de nitrogênio na seiva do tomateiro por meio de microeletrodo portátil. Horticultura Brasileira, Brasília, 16(2):144-150, 1998. 
GRATANI, L. A non-destructive method to determine chlorophyll content of leaves. Photosynthetica, Flemingovo, 26(4):469-473, 1992.

JACKSON, M.L. Analisis químico de suelos. Barcelona, Ediciones Omega, 1982. 662 p.

LOPEZ-CANTARERO, I.; LORENTE, F. \& ROMERO, L. Are chlorophylls good indicators of nitrogen and phosphorus levels? Journal of Plant Nutrition, New York, 17(6):979990, 1994.

MINOTTI, P.L.; HALSETH, D.E. \& SIECZKA, J.B. Field chlorophyll measurements to assess the nitrogen status of potato varieties. HortScience, Minnesota, 29(12):14971500, 1994.

PIEKIELEK, W.P. \& FOX, R.H. Use of a chlorophyll meter to predict sidedress nitrogen requirements for maize. Agronomy Journal, Madison, 84(1):59-65, 1992.
SCHEPERS, J.S.; FRANCIS, D.D.; PVCGIL, M. \& BELOW, F.E. Comparison of corn leaf-nitrogen concentration and chlorophyll meter readings. Communications in Soil Science and Plant Analysis, New York, 23(17/20):21732187, 1992.

SHADCHINA, T.M. \& DMITRIEVA, V.V. Leaf chlorophyll content as a possible diagnostic mean for the evaluation of plant nitrogen uptake from the soil. Journal of Plant Nutrition, New York, 18(7):1427-1437, 1995.

SMEAL, D \& ZHANG, H. Chlorophyll meter evaluation for nitrogen management in corn. Communications in Soil Science and Plant Analysis, New York, 25(9/10):14951503, 1994.

YADAVA, U.L. A rapid and nondestructive method to determine chlorophyll in intact leaves. HortScience, Minnesota, 21(6):1449-1450, 1986. 